\title{
A Rare Case of Synchronous Ovarian Adenocarcinoma and Squamous Cell Carcinoma of Cervix
}

\author{
Logeswary Nadarajan, Lee Saw Joo, and Zalina Nusee
}

\section{ABSTRACT}

Synchronous tumours of gynaecological malignancies occur rarely and most of these cases are represented by synchronous ovarian and endometrial cancer. Synchronous malignancies of cervix and ovary are rare with poor prognosis. Only few cases of synchronous cancer of cervix and ovary are found in the literature as case reports. Here, we report a case of a 63 year old patient who was diagnosed with synchronous squamous cell carcinoma of cervix and high grade serous carcinoma of ovary in which her clinical presentation, investigation and intraoperative findings were atypical. Patient presented with postmenopausal bleeding and mass per abdomen. Pipelle sampling revealed squamous cell carcinoma of cervix. Examination under anaesthesia noted endocervical growth measuring $3 \times 4 \mathrm{~cm}$ with endoluminal extension into the whole endometrial cavity. Computerized tomography (CT) imaging showed left ovarian mass measuring $10.0 \times 11.7 \mathrm{~cm}$. Uterine corpus involvement in this case mislead us to the initial diagnosis of ovarian metastasis in cervical cancer. The distinct histopathological features of the ovary, cervix and endometrial lesion post operatively helped in establishing the diagnosis of two separate primaries which was synchronous cervical and ovarian cancer rather than metastatic spread of one primary malignancy.

Keywords: Metastatic lesions; synchronous tumours; squamous cell carcinoma of cervix; serous carcinoma of ovary.

\section{INTRODUCTION}

Synchronous gynaecological malignancies are rare with overall incidence of $0.63 \%$ [1], [2]. About 1-2\% of all women with gynaecological cancers have two or more simultaneous independent primary tumours involving female genital tract [4]-[6]. Low stage and low grade synchronous tumours must be distinguished from metastatic tumours for accurate management as they carry better prognosis compared to metastatic counterpart. Risk factors for individual types of synchronous tumours are varying with the nature of the tumour. The mechanism of synchronous cancers remains unclear, but the most popular hypothesis is the similar embryologic origin or a common cell line [7]. The most common combination is those of the ovary and endometrium constitute $40 \%$ of all synchronous tumours [3]. Synchronous cervical and ovary cancer is rare in literature and only found in few case reports. Cervical cancer rarely metastasizes to the ovaries. The proportion of cases presenting with ovarian metastasis at the time of surgery ranges from $0.6-1.5 \%$ [8]. Adenocarcinoma of cervix compared to cervical squamous cell type metastasis more common to ovary with incidence of $0.6-1.5 \%$ [8]. The aim of this report is to present the case of a patient who was initially diagnosed with cervical squamous cell carcinoma
Submitted : March 26, 2021

Published : April 21, 2021

ISSN: 2593-8339

DOI: $10.24018 /$ ejmed.2021.3.2.783

\section{Logeswary Nadarajan *}

Department of Obstetrics \& Gynaecology, SASMEC@ IIUM,

Malaysia.

(e-mail: loges.nadarajan@yahoo.com)

Lee Saw Joo

Department of Obstetrics \& Gynaecology, Hospital Raja

Permaisuri Bainun, Malaysia.

(e-mail: leesawjoo@ gmail.com)

Zalina Nusee

Department of Obstetrics \& Gynaecology, SASMEC@ IIUM, Malaysia.

(e-mail: drzalina@iium.edu.my)

*Corresponding Author

with ovarian metastasis based on clinical and intraoperative findings however the final diagnosis was synchronous cervical and ovarian cancer from histopathology report. Patient was investigated for post menopausal bleeding which revealed squamous cell carcinoma of cervix and imaging showed ovarian mass. Examination under anaesthesia revealed endocervical growth with uterine corpus involvement. This made us to come up with impression of metastasis of cervix to ovary. Patient underwent neoadjuvant chemotherapy followed by Wertheim's hysterectomy, bilateral salphingoopherectomy, omentectomy and appendicectomy with debulking. Histopathology result of benign lesion of the endometrial poly and distinct histological features of cervix and ovary brought to the final diagnosis of synchronous tumour of cervix and ovary. However, it could still be a synchronous tumour of cervical and endometrium. This is if the endometrial polyp which originally could had been a carcinomatous serous endometrial polyp with metastasis to ovary prior to initiation of neoadjuvant chemotherapy. 


\section{CASE REPORT}

Patient is a 63 year old postmenopausal woman, para 3 with diabetes mellitus presented with complaints of abdominal pain for four months. Further questioning revealed patient to have on and off fever and foul smelling per vaginal bleeding for the past 2 weeks. Patient did not notice any mass per abdomen and no constitutional symptoms. Bowel habit was remaining same and no urinary symptoms. Cervical screening was not done previously. Significant clinical examination was per abdomen noted vague mass palpable over left lower abdomen which was located at POD on vaginal examination. It was fixed, irregular and tender on pressure. Pelvic examination showed normal ectocervix, however bulky endocervix.

Trans-abdominal scan noted atrophic uterus with endometrial thickness of $14 \mathrm{~mm}$. Right complex adnexae mass measuring $8.2 \times 8.8 \mathrm{~cm}$, mixed solid cystic in nature with no papillary projections and septations. Heterogenous cervical mass measuring $3 \times 4 \mathrm{~cm}$. No ascites and no hydronephrosis.

Tumour marker cancer antigen 125 was 240 International unit (IU). Pipelle endometrial sampling taken to investigate for postmenopausal bleeding revealed high grade dysplasia of squamous epithelium with foci of microinvasion.

Patient underwent examination under anaesthesia which revealed presence of endocervical growth $3 \times 4 \mathrm{~cm}$ with endoluminal extension into the whole endometrial cavity on hysteroscopic finding. Left parametrium thickened with left adnexal mass $10 \times 10 \mathrm{~cm}$ fixed to uterus. Biopsy taken from endocervical growth showed squamous cell carcinoma.

On CT imaging, there was a complex mass between the uterus and rectosigmoid colon measuring $10.0 \times 11.7 \mathrm{~cm}$. The mass was heterogeneously enhanced with mixed solid-cystic component. Demarcation with uterine cervix and rectum is obliterated. Uterus was pushed anteriorly by the mass and no clear plane of demarcation with the mass. Minimal hydrometra in the uterine cavity and no uterine mass seen. The uterine cervix appears prominent measuring 4.0×2.6 $\mathrm{cm}$. Minimal ascites present and multiple para-aortic nodes enlargement.

At this point, the diagnosis was squamous cell carcinoma of cervix with ovarian metastasis and nodal metastasis. Patient was subjected to chemotherapy with three cycles cisplatin and paclitaxel. Repeat CT imaging post neoadjuvant chemotherapy showed resolved nodal metastasis and tumour shrinkage.

Subsequently patient underwent Wertheim's hysterectomy with bilateral salphingoopherectomy, omentectomy, appedicectomy and debulking. Intraoperatively noted left ovarian tumour $8 \times 8 \mathrm{~cm}$ and encapsulated. Uterus was dissected, revealed previous cervical mass over the endocervix responded to chemotherapy. In the endometrium, presence of polypoidal growth noted over fundus of uterus. Histopathological report showed residual squamous cell carcinoma within endocervix $1 \mathrm{x} 1 \mathrm{~cm}$ with left ovarian high grade serous adenocarcinoma $9 \times 7 \times 5 \mathrm{~cm}$. The polypoidal lesion in the endometrium were benign endometrial polyp.

Final diagnosis was synchronous squamous cell carcinoma of cervix stage $2 \mathrm{~B}$ with left ovarian high grade serous adenocarcinoma stage 2 .
Postoperatively patient completed another three cycles of adjuvant chemotherapy. Repeated CT imaging showed no metastasis and patient had been on follow up for two years with disease free.

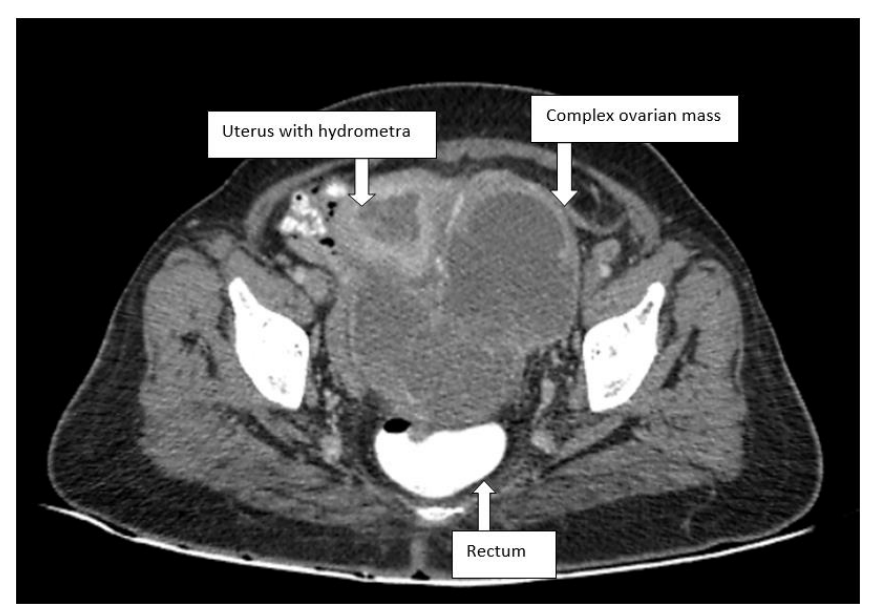

Fig. 1. CT imaging findings of left complex ovarian mass with solid cystic component and demarcation with the uterine cervix and rectum obliterated.

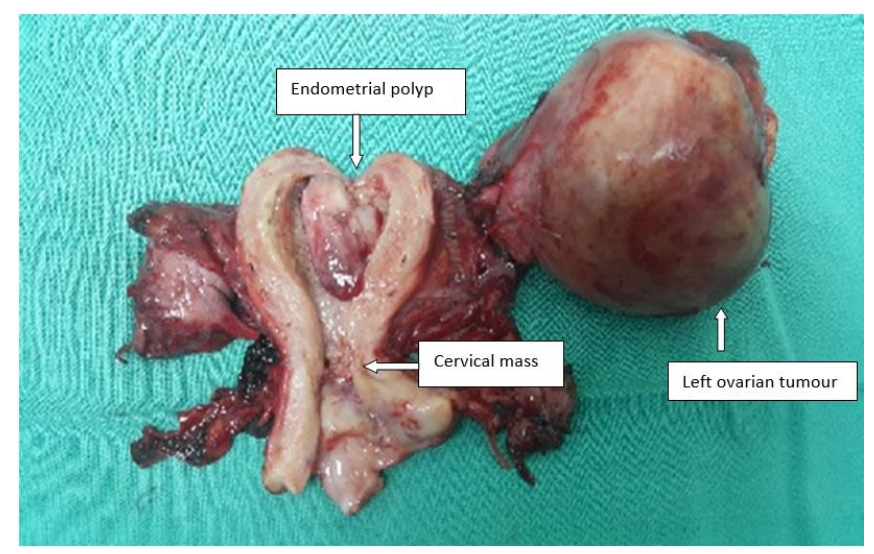

Fig. 2. Specimen of uterus and left ovarian tumour with cervical mass responded to chemotherapy (over endocervix) with polypoidal growth over fundus of the uterus.

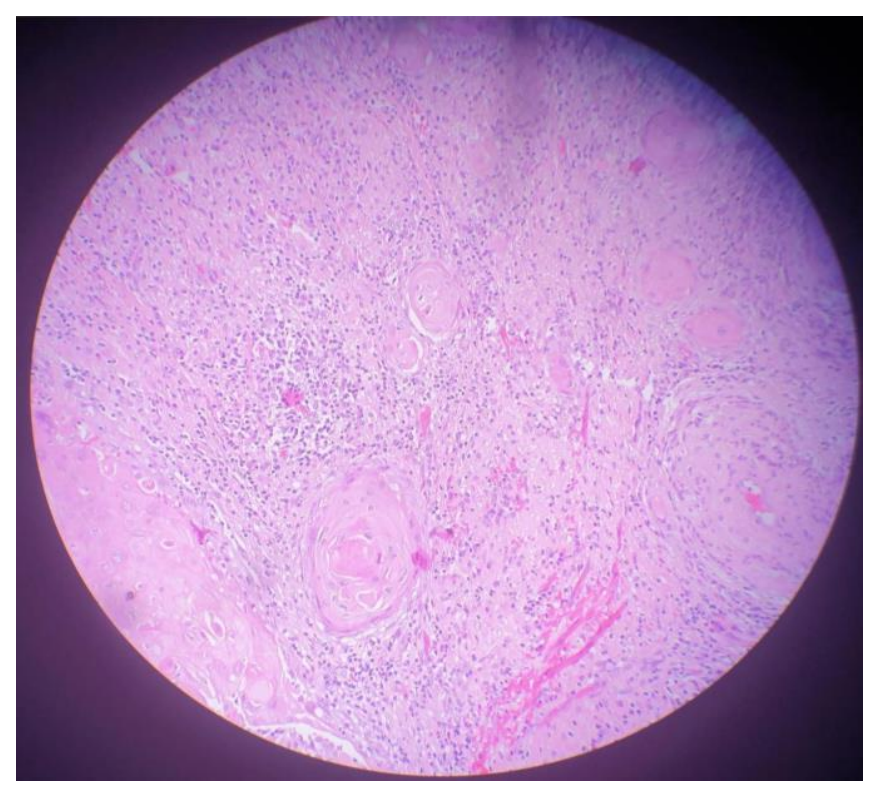

Fig. 3. Histopathological picture of cervical tumour shows nests and clusters of moderately differentiated squomous epithelium infiltrating into the stroma. 


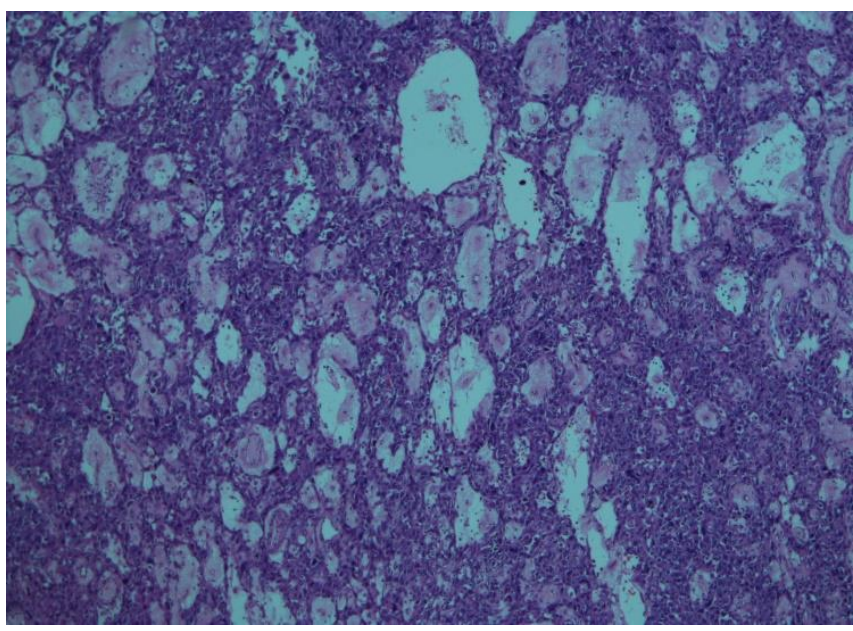

Fig. 4. Histopathology picture of high grade serous adenocarcinoma of ovary with the malignant cells exhibit pleomorphic and hyperchromatic nuclei.

\section{DISCUSSION}

The foundation and stimulus for study on multiple primary malignant tumour (MPMT) was provided by Billroth [9] in 1889. He pointed out that not only is it possible for a patient to have two simultaneous developing independent cancer, but it is also possible for a second primary cancer to develop in cancer prone individual after an initial malignant lesion has been treated [10]. MPMTs may be defined as synchronous or metachronous tumors. "Synchronous" tumors refer to cases in which the second primary cancer is diagnosed within 6 months of the primary cancer; "metachronous" tumors refer to cases in which the second primary cancer is diagnosed more than 6 months after the diagnosis of the first primary cancer [11]. Synchronous gynaecological malignancies have been encountered in up to $2 \%$ of women diagnosed with any kind of gynaecological cancer. The most commonly encountered association consist of ovarian and endometrial cancer which accounts for $50-70 \%$ of all synchronous female genital tract tumour [4]-[6]. As for the association between ovarian and cervical cancer only isolated cases have been reported so far.

In such cases the most important thing to determine is if the two lesions are really synchronous, distinct malignancies, or one of them is the metastasis of the other tumour. This fact can be clearly ruled out in cases in which the tumours exhibit different histological subtypes, as well as in cases in which areas of normal parenchyma can be evidenced between the two lesions. In our case, the presence of two different histopathological subtypes (high grade serious carcinoma of ovary and squamous cell carcinoma of cervix) making diagnosis of synchronous lesions an indubitable one.

In contrast to cervical cancer with ovarian metastasis, in synchronous type, the endocervical primary is entirely intraepithelial or shows minimal subtle foci of invasion. In this case, there were no lymphovascular or neural invasion and the ectocervix and endocervical glands were unremarkable. Distinction of primary ovarian tumors from metastatic tumors is often challenging, not only at the time of pre-operative assessment, but also during intra-operative assessment required for staging decision. Previous studies have shown that a simple algorithm using tumor size and laterality can accurately classify a substantial majority of tumors [12]. Metastatic ovarian tumour has been typically described to be bilateral, $<100 \mathrm{~mm}$ in diameter with characteristics involvement of ovarian surface with ovarian parenchyma spared [12]. Meanwhile those of primaries are typically large, unilateral and tumour arise from within ovarian epithelium as in our case.

It is recognized that adenocarcinoma of the cervix metastasizes to the ovaries with greater frequency than squamous cell carcinoma [13]. Prognosis in concurrent tumours is relatively good especially with endometrium or cervix carcinoma reason being concurrent ovary tumours can be diagnosed in early stage [14]. Symptoms in cervix and endometrium generally appear early while ovarian cancers are generally asymptomatic during early stage. In this case, patient presented to hospital with complaint of post menopausal bleeding however further examination and investigations revealed ovarian mass and lead to earlier diagnosis of ovarian carcinoma. Our initial diagnosis was supported by a study by Kim et al [15] aimed at investigating various risk factors for ovarian metastasis in cervical cancer including age, histologic types, stromal invasion, FIGO stages, lymph node metastasis, parametrial invasion, involvement of the upper vagina and uterine corpus, concluded that uterine corpus involvement in addition to histologic type were the independent risk factors for ovarian metastasis. Transtubal implantation has been postulated as a mechanism of spread. However, it had turned out that we were misleaded in our patient by an endometrial lesion that eventually turned out to be benign endometrial polyp which originally could had been a carcinomatous serous endometrial polyp prior to initiation of chemotherapy. Unfortunately, in our case the endometrial lesion was not sampled prior to neoadjuvant treatment and this had been the learning point of our case report. The treatment strategies for synchronous cancers depend on the established strategy for each individual cancer and, both tumors may be treated surgically with good prognosis. Cases of cervical cancer with ovarian metastasis usually present in advanced stage. Management differs with stage at presentation and mostly will be subjected to concurrent chemoradiotherapy post operatively and carries poor prognosis.

Molecular testing and techniques such as gene expression profiling, beta-catenin gene mutational analysis, pTEN has been advocated for valuable adjunctive information in ambiguous cases. Pertaining to synchronous ovarian and cervical carcinoma, role of p16 and HPV analysis by in situ hybridization or polymerase chain reaction is recommended to identify cases of synchronous and metastasis [16].

\section{CONCLUSION}

Synchronous genital tract neoplasms are more clinically complex compared with single neoplasms and it is crucial to focus on the differential diagnosis between primary and metastatic tumors during the diagnostic process as the management of each entity differ and the prognosis too.

Although rarely seen, synchronous cervical and ovarian cancer might be encountered and be beneficial from surgery with curative intent. 


\section{REFERENCES}

[1] Srivastava K, Zahra F. Synchronous primary malignancy of ovary and cervix with different histopathology: A rare presentation. The Internet Journal of Gynecology and obstetrics 2009; Volume 12 Number 2.

[2] Kambi DP, Mallikarjuna M, Santosh C, Abhishek V. Synchronous malignancies of ovary, fallopian tube and cervix: A rare case. International Journal of Biomedical and Advance Research 2013; 4(9): 676-679.

[3] RD Ratke, Shruti Gadekar, Priyanka Pagare. A Rare Case of Carcinoma of Ovary with Carcinoma of Cervix. Journal of Case Report 2014;4(1):217-220.

[4] Ayhan A. Yalchin OT, Tuncer ZS, Gargan T, Kucukali T. Synchronous primary malignancies of the female genital tract. Eur. J. Obstet. Gynecol. Repro. Biol. 1992: 45; 63-66.

[5] Eisner RF. Nieberg RK. Berek JS. Synchronous primary neoplasm of the female reproductive tract. Gynecol. Oncol.1989:33;335-339.

[6] Tong SY. Lee YS. Park JS. Bae SN. Lee JM. Namkoong SE. Clinical analysis of synchronous primary neoplasms of the female reproductive tract.Gynecol.Reprod.Biol.2008:136;78-82.

[7] Garcia SB, Novelli M, Wright NA. The clonal origin and clonal evaluation of epithelial tumours. Int J ExpPathol.2008;81:89-116.

[8] Shimada M, Kigawa J, Nishimura R, Yamaguchi S, Kuzuya K, Nakanishi T, Suzuki M, Kita T, Iwasaka T, Terakawa N. Ovarian metatstasis in carcinoma of the uterine cervix. Gynecol Oncol.2006 May;101(2): 234-7. doi:10.1016/j.ygyno.2005.10.004.Epup2005 Nov 21.PMID: 16300819.

[9] Billroth, T: Die allgemeine chirurgische Pathologie und Therapie in 51 Vorlesungen; ein Handbuch für Studirende und Ärtze, 14. Aufl. Berlin, Germany. G. Reimer. 1889; p.908.

[10] Charles G Moertel, Malcolm B Dockerty, Archie H Baggentoss. Multiple primary malignant neoplasm. I. Introduction and presentation of data. Cancer 14(2), 221-230, 1961. acsjournals.onlinelibrary.Wiley.com.

[11] Chongya Zhai, Yulan Cai, Fang Lou, Zhen Liu, Jiansheng Xie, Xiaoyum Zhou, Zhanggui Wang, Yong Fang, Hongming Pan, Weidong Han. Multiple primary malignant tumours-A clinical analysis of 15,321 patients with malignancies at a single centre in China. Journal of cancer. 2018; 9(16): 27952801.doi:10.7150/jca.25482.

[12] Yemelyanova AV, Vang R, Judson K, Wu LS, Ronnett BM. Distinction of primary and metastatic mucinous tumours involving the ovary: analysis of size and laterality data by primary site with reevaluation of an algorithm for tumor classification. AM. J. Surg. Pathol. 2008; 32; 128-138.

[13] Singh N, Arif S. Histopathologic parameters of prognosis in cervical cancer-a review. Int. J. Gynecol. Cancer 2004;14;741-750.

[14] Qadir Fatima, Sonam Dubey, Suman Kapuriya. Synchronous primary carcinomas of cervix and ovary: A case report. IOSR Journal of Dental and Medical Sciences (IOSR-JDMS) e-ISSN:22790861.Volume15, Issue 8 Ver. V(August.2016), PP71-75.

[15] Kim MJ, Chung HH, Kim JW, Park NH, Song YS, Kang SB. Uterine corpus involvement as well as histologic type is an independent predictor of ovarian metastasis in uterine cervical cancer. J Gynecol Oncol $2008 \quad$ Sep;19(3). 181-184 doi:10.3802/jgo.2008:.19.3.181[PMC free article] [PubMed].

[16] Vang R, Gown AM, Farinola M et al, p16 expression in primary ovarian mucinous and endometriod tumors and metastatic adenocarcinomas in the ovary: utility for identification of metastatic HPV-related endocervical adenocarcinomas. Am. J. Surg. Pathol. $2007 ; 31 ; 653-663$. 